\title{
Queratoquiste odontogénico: reporte de un caso.
}

\section{Odontogenic keratoquistic: a case report.}

\author{
Andrade-Barahona Celena ${ }^{1}$, Torres-Calle Fernanda ${ }^{2}$, Pineda-Álvarez David $^{3}$, Viteri-Luzuriaga Luis $^{4}$, \\ Heras-Chávez Jessica ${ }^{5}$. \\ ${ }^{1}$ Rotante de Investigación en la Universidad Católica de Cuenca, Ecuador. Odontóloga de práctica privada. ORCID iD: \\ https://orcid.org/0000-0002-0865-7336. \\ ${ }^{2}$ Esp. en Patología Bucomáxilo Facial. Docente de la Universidad de Cuenca, Ecuador. ORCID iD: \\ https://orcid.org/0000-0002-8097-212x \\ ${ }^{3}$ Esp. en Cirugía Oral y Maxilofacial. Mg. en Investigación de la Salud. Docente de la Universidad de Cuenca, Ecuador. \\ ORCID iD: https://orcid.org/0000-0002-6395-7702 \\ ${ }^{4}$ Esp. en Cirugía Oral y Maxilofacial. Docente de la Universidad Católica Santiago de Guayaquil, Ecuador. ORCID iD: \\ https://orcid.org/0000-0001-5050-2701 \\ ${ }^{5}$ Rotante de Investigación en la Universidad Católica de Cuenca, Ecuador. Odontóloga de práctica privada. \\ * celena.andradeb22@ucuenca.ec
}

\begin{abstract}
Resumen
El queratoquiste odontogénico es una lesión benigna de los maxilares de alta prevalencia y de naturaleza agresiva, que afecta principalmente a personas de la segunda, tercera y cuarta década de vida. El queratoquiste posee una mayor predilección por el sexo masculino en una proporción de 2: 1 y es más frecuente que se desarrolle en la mandíbula que en el maxilar. Generalmente esta lesión es asintomática, aunque en algunos casos suele haber dolor y expansión de las corticales óseas. Radiográficamente se presentan como radiolucencias uniloculares o multiloculares e histológicamente su característica principal es su revestimiento de epitelio escamoso estratificado paraqueratinizado. Esta lesión tiene una alta tasa de recurrencias que van desde el $5 \%$ hasta el $70 \%$ y a pesar de que se han realizado varios estudios sobre la mejor opción de tratamiento para evitar las reincidencias, todavía no se ha encontrado un procedimiento que sea estadísticamente mejor que otro. En el presente reporte se muestra un caso de queratoquiste odontogénico con sus características imagenológicas e histopatológicas, su tratamiento y el seguimiento tras un año posoperatorio.
\end{abstract}

Palabras clave: Queratoquiste, quiste, tumor, odontogénico, curetaje.

\begin{abstract}
The odontogenic keratocyst $(O K C)$ is a benign lesion of the jaws with high prevalence and aggressive nature, which mainly affects people of the second, third and fourth decade of life. The keratocyst has a greater predilection for male sex 2:1 ratio and is more frequent to develop in the jaw than in the maxilla. Generally, this lesion is asymptomatic although in some cases there is usually pain and expansion of the cortical bones. Radiographically, they appear as unilocular or multilocular radiolucences. Histologically their main feature is a parakeratinized stratified squamous epithelium lining. This lesion has a high recurrence rate ranging from 5\% to $70 \%$. Although several studies have been conducted on the best treatment option to prevent recurrence, a procedure that is statistically better than another has not yet been found. The present report shows a case of odontogenic keratocyst with its imaging and histopathological characteristics, its treatment and follow-up after a postoperative year.
\end{abstract}

Key words: Keratocyst, cyst, tumor, odontogenic, curettage.

\section{INTRODUCCIÓN}

El queratoquiste odontogénico (Odontogenic Keratocyst $(\mathrm{OKC})$ ) es una lesión benigna de los maxilares de alta prevalencia y de naturaleza agresiva. En el año 2005 la Organización Mundial de la Salud (OMS) clasificó al queratoquiste odontogénico como tumor odontogénico queratoquístico; sin embargo, se consideró que no existía evidencia suficiente para catalogarlo como una neoplasia y en el año 2017 la OMS actualizó su cuarta edición de la Clasificación Mundial de Tumores de Cabeza y Cuello: tumores óseos odontógenos y maxilofaciales, en la que al tumor odontogénico queratoquístico se lo reconoció nuevamente dentro de la categoría de quiste como: queratoquiste odontogénico en base a criterios 
como su crecimiento agresivo, su alta tasa de recurrencia postratamiento, la rara ocurrencia de una variante sólida de OKC y las mutaciones en el gen PTCH. ${ }^{1}$

\section{ETIOPATOGENIA}

Se ha demostrado que el OKC puede originarse de los restos de las células basales del epitelio oral, del órgano dental por degeneración del retículo estrellado, o del remanente epitelial odontogénico de los maxilares., ${ }^{2,3}$ Existen tres teorías que explican la causa del desarrollo del OKC: un incremento de la replicación de las células basales, aumento de la presión osmótica, y liberación de factores de reabsorción ósea que inducen la actividad enzimática osteolítica. $^{2}$

\section{CARACTERÍSTICAS CLÍNICAS}

El OKC corresponde al 4-12\% de los quistes odontogénicos. Existe una mayor prevalencia entre la segunda, tercera y cuarta década de vida, ${ }^{4}$ sin embargo, posee un amplio rango de edad, pudiendo manifestarse desde la primera hasta la octava década de vida, ${ }^{2}$ aunque la presentación en niños es muy rara. ${ }^{4}$ En cuanto a su predilección por sexo tiene una mayor inclinación por el sexo masculino en una proporción de $2: 1 .^{2}$ Es más frecuente que el OKC se desarrolle en la mandíbula que en el maxilar. En el cuerpo y la rama mandibular se ha observado en un $60 \%$ de los casos, mientras que en la región posterior del maxilar se ha contemplado que sucede en un $21,6 \%$, en la zona anterior del maxilar en un $8,5 \%$ y en menor frecuencia en el mentón en un $7 \%$; no obstante, se han reportado casos de OKC en ubicaciones periféricas como en el seno maxilar, ${ }^{5}$ y en el hueso temporal. ${ }^{6}$

Si bien es cierto, el quiste generalmente carece de sintomatología y se lo evidencia a partir de hallazgos radiográficos, ${ }^{2,7}$ en ocasiones, al infectarse pueden generar dolor o inflamación de los tejidos circundantes, ${ }^{4}$ y cuando existen infecciones secundarias si se perforan las corticales óseas y los tejidos que yacen sobre ellas. ${ }^{8}$ Además Rajendra y Berge mencionan que es infrecuente que el OKC genere desplazamiento de los dientes o expanda el hueso cortical, ${ }^{4,8}$ pero eventualmente tiende a generar reabsorción intramedular ósea. ${ }^{8} \mathrm{El}$ mecanismo por el cual el queratoquiste se dilata se debe al aumento en el recambio epitelial, a diferencia de otras lesiones quísticas de los maxilares que se expanden por aumento de la presión osmótica. ${ }^{9}$ Radiográficamente, se muestra como radiolucencias uniloculares o multiloculares, ${ }^{4,10}$ con bordes corticalizados ya sean definidos o festoneados. ${ }^{2,10}$

El OKC puede manifestarse como una lesión única o de manera múltiple asociado al carcinoma basocelular nevoide
(NBCCS) o síndrome de Gorlin Goltz, ${ }^{11}$ que es una rara enfermedad autosómica dominante ocasionada por mutaciones en el gen PTCH ubicado en el cromosoma 9q. ${ }^{1,2,12}$ Sin embargo, Zecha en el $2010^{11}$ estableció que no necesariamente la presencia de múltiples OKC implican que un paciente sufra del síndrome NBCCS; más bien se refiere al historial de vida del paciente.

\section{CARACTERÍSTICAS HISTOLÓGICAS}

Microscópicamente el OKC está revestido por una superficie corrugada de un epitelio escamoso estratificado paraqueratinizado, siendo esta característica el criterio para su diagnóstico histopatológico. ${ }^{4,8,11}$ El epitelio queratinizado consta de 6 a 11 células de espesor que mantienen su núcleo en las capas más externas. La capa basal expone células columnares altas con sus núcleos en empalizada, polarizados, de diámetro uniforme, e intensamente teñidos. La separación entre el epitelio y la cápsula es plana y dentro del tejido conectivo se observa un infiltrado inflamatorio crónico, con contenido de líquido o queratina, aunque se han informado lesiones de naturaleza sólida. ${ }^{4,8}$

\section{PRESENTACIÓN DEL CASO}

Paciente de sexo femenino, de 24 años de edad, fue referida por su ortodoncista al cirujano maxilofacial, luego de haber observado en las radiografías con fines diagnósticos previas al tratamiento ortodóncico, una lesión radiolúcida bien circunscrita en la rama mandibular izquierda relacionada a la pieza 3.8 que se encontraba retenida (Figura 1).

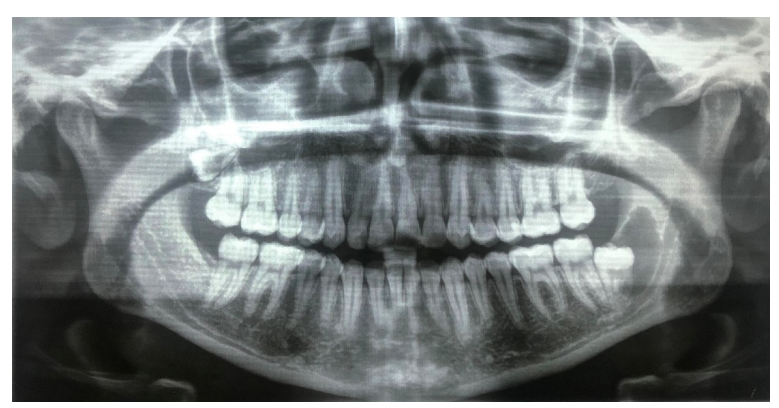

Fig. 1. Radiografía panorámica inicial. Nótese el área radiolúcida bien definida en rama mandibular izquierda circunscrita a la pieza 3.8 retenida.

La paciente no evidenciaba sintomatología, al examen extraoral no existía asimetría facial y al examen intraoral no se observaba expansión de las corticales óseas y los tejidos blandos mostraban características normales. Se solicitó una tomografía computarizada complementaria a las imágenes radiográficas (Figura 2) y posteriormente se procedió a la cirugía. 


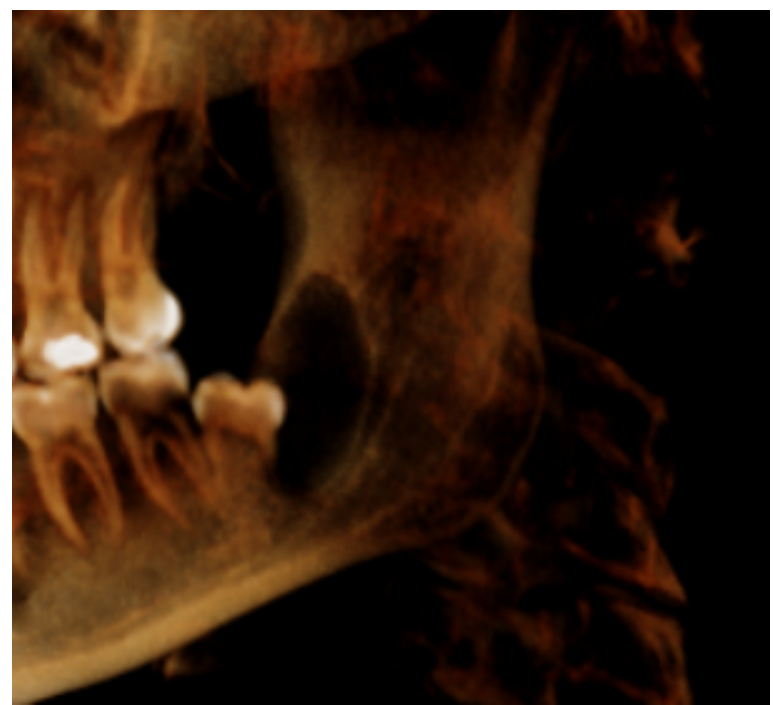

Fig. 2. Tomografía computarizada. Se observa área radiolúcida abarcando la rama mandibular izquierda y adosada a pieza 3.8.

El procedimiento fue ejecutado de manera ambulatoria bajo anestesia local, se retiró el quiste mediante enucleación, se extrajo la pieza comprometida y se realizó el curetaje del lecho quirúrgico, eliminando también una pequeña porción del trabeculado óseo circundante. Durante el acto quirúrgico además se logró evidenciar clínicamente el paquete vasculonervioso alveolar inferior el cual se lo mantuvo intacto. Posteriormente se efectuó un lavado riguroso de la zona con solución salina estéril; no se usó ninguna sustancia química o cáustica y los tejidos no fueron cauterizados. Para la regeneración ósea se utilizaron $2 \mathrm{cc}$ de Aloinjerto Particulado Cortical Puros $₫$ (Zimmer Biommet) y plasma rico en fibrina (PRF) obtenido de la misma paciente minutos antes de la cirugía. La lesión se colocó en formalina y fue enviada al laboratorio para el análisis histopatológico.

Previo a realizar el estudio histopatológico de la lesión, esta se la separó de la pieza dental mediante un bisturí para su análisis macroscópico. Se encontró un tejido de forma alargada, color blanquecino, de superficie regular y consistencia firme, que medía $1,9 * 1,4 * 1,1 \mathrm{~cm}$ (Figura 3).

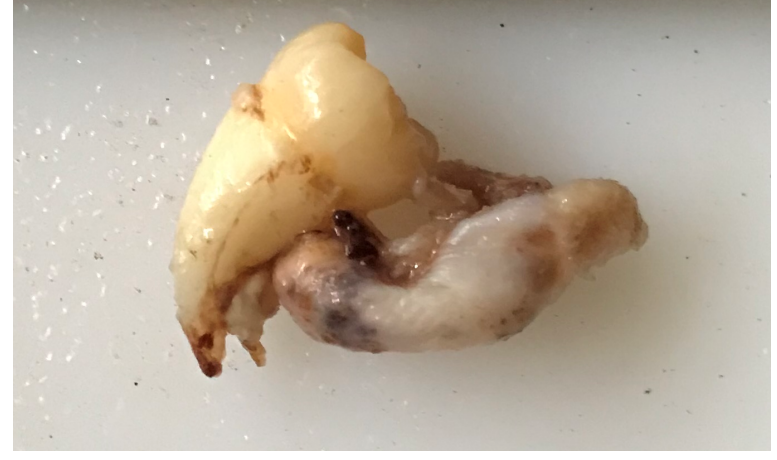

Fig. 3. Macroscopía. Tejido blando adosado a la pieza 3.8.

En el análisis microscópico se encontró material eosinófilo compatible con queratina descamada rodeada por epitelio plano estratificado paraqueratinizado, cuyas células de la capa basal se mostraron cúbicas, hipercrómaticas, y con sus núcleos dispuestos en polarización reversa; además este epitelio se manifestó hiperplásico en tramos. Dentro del tejido conectivo que forma la cápsula de la lesión se observó la presencia de zonas con abundante infiltrado inflamatorio crónico con predominio de plasmocitos y macrófagos, así como depósitos intracelulares de colesterol (macrófagos espumosos) y depósitos extracelulares generados por la ruptura de las células que contienen el colesterol (cristales de colesterol) (Figura 4 y 5).

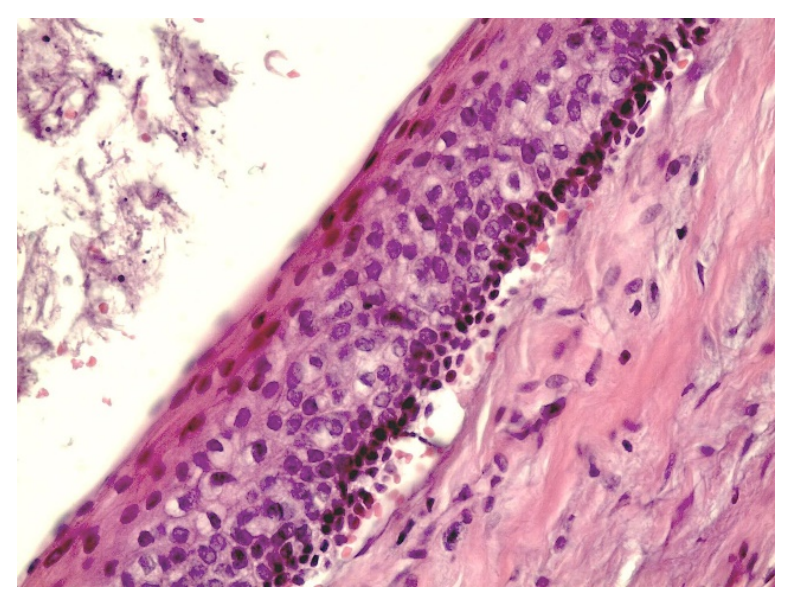

Fig. 4. Microscopía. Queratina descamada y epitelio plano estratificado paraqueratinizado, depapilado, células de la capa basal hipercromáticas H\&E, 40x. 


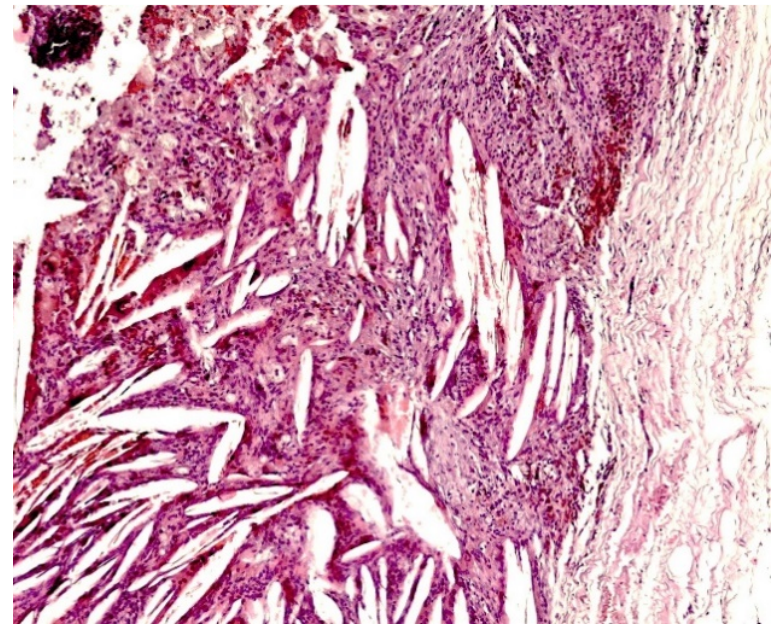

Fig. 5. Microscopía. Cristales de Colesterol H\&E, 100X

Al observar el control radiográfico luego de un año de haber realizado el tratamiento quirúrgico, se evidenció aumento de la radiopacidad a nivel de la rama mandibular y regeneración del trabeculado óseo sin aparición de posibles recurrencias o lesiones satélites (Figura 6).

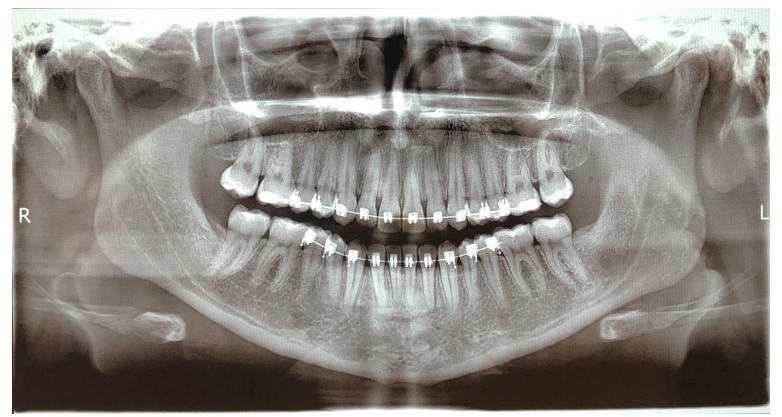

Fig. 6. Radiografía panorámica de control. Aspecto radiográfico postoperatorio de la mandíbula después de 1 año del tratamiento.

\section{DISCUSIÓN}

El queratoquiste odontogénico es considerado como una lesión de comportamiento agresivo por su naturaleza de expansión dentro del hueso. ${ }^{8}$ Al tener características clínicas y radiográficas muy similares a varias lesiones de los maxilares como el quiste dentígero, ameloblastoma, quiste periodontal lateral, quiste radicular, tumor odontogénico adenomatoide, fibroma ameloblástico, granuloma central de células gigantes y mixoma odontogénico, ${ }^{2,4,13,14}$ es fácil confundir al OKC con estos trastornos, por lo tanto, su diagnóstico definitivo es histopatológico.

Todavía no se ha llegado a un conceso sobre el tratamiento más adecuado, sin embargo, se presentan dos opciones de tratamiento: conservador y agresivo., 30
Varios estudios coinciden con el procedimiento habitual conservador para tratar los $\mathrm{OKC}$ pequeños, que consiste en su enucleación quirúrgica y en cuanto a las lesiones de mayor tamaño se opta por el mismo proceso, pero con marsupialización o descompresión previa de la misma. ${ }^{3,8,10,11,15}$ El tratamiento agresivo incluye resección quirúrgica u ostectomía periférica. ${ }^{3,4,9-11}$ Se han propuesto varios tratamientos complementarios adicionales como el legrado químico. Stoelinga en el año 2005, ${ }^{16}$ realizó un estudio sobre la enucleación de la lesión con la posterior colocación de la solución de fijación de Carnoy en la cavidad y Schmidt en año $2003^{17}$ manifestó el uso del nitrógeno líquido para crioterapia o congelación, ambos métodos actúan sobre el tejido residual y generan un legrado óseo para prevenir la recurrencia de la lesión. ${ }^{8,9}$ La solución de Carnoy tiene la ventaja de cauterizar las células tumorales; sin embargo, un notable efecto adverso es la posibilidad de daño local al nervio ocasionando parestesia o paresia. A pesar de ello, esta terapia ha tenido éxito en la mayoría de los casos. El nitrógeno líquido se ha usado gracias a su capacidad de desvitalizar el hueso en el área de la lesión a través de la formación de cristales de hielo dentro y alrededor de las células, dejando intacta una capa delgada de tejido inorgánico. $^{2}$

En este caso el tratamiento fue la enucleación completa de la lesión y se complementó con legrado óseo a través de un curetaje manual, sin usar ningún tipo de solución química. Al realizar el control luego de 12 meses después de la cirugía, se constató la formación e integración de hueso en la región donde se encontraba el quiste. Estos resultados son semejantes a los del caso publicado por Sembronio en el año 2009, ${ }^{15}$ en el que, para eliminar un queratoquiste, se utilizaron instrumentos para hacer un raspado en el defecto óseo y después de 3 años de seguimiento no se observó evidencia alguna de reincidencias ni clínicas ni radiográficas. O’Neill en el año $2011,{ }^{18}$ en su publicación, se realizó un procedimiento similar para tratar un OKC: enucleación simple de la lesión y decorticalización de las paredes óseas a través de una fresa redonda No. 8 con la colocación posterior del aloinjerto de Puros (Zimmer, Carlsbad, CA) y una membrana reabsorbible Ossix (OraPharma, Warminster, PA) para rellenar y cubrir la cavidad residual en el hueso; en el control radiográfico un año después de la cirugía se evidenció la resolución completa de la lesión.

Es importante mencionar que la escisión quirúrgica del OKC debe hacerse de la manera más cuidadosa posible debido a que el revestimiento del quiste es muy delicado y frágil y su recurrencia es causada por una eliminación insuficiente del revestimiento de la lesión, de los restos epiteliales, de la capa de células basales del epitelio oral, y de una asociación con el síndrome de Gorlin Golz. ${ }^{2-4}$ Además de las determinantes mencionadas para la reaparición 
de las lesiones queratoquísticas, la recurrencia está dada también por el crecimiento de nuevas islas epiteliales o quistes satelitales pequeños en una región próxima. ${ }^{2,3,16}$ No obstante, sin importar el origen epitelial para la reaparición del OKC, los microquistes se han encontrado adheridos a la mucosa que rodea el quiste en aproximadamente el $50 \%$ de los casos. ${ }^{16,19}$

El queratoquiste presenta una tasa de recurrencia muy variante que va desde reincidecias bajas del $5 \%$ hasta muy altas llegando hasta un $70 \%$. $, 8,10,14$ Se ha informado que la mayor parte de las recurrencias ocurren dentro de los primeros cinco años postratamiento, aunque se han reportado apariciones tardías hasta 40 años después del tratamiento inicial. ${ }^{19}$ Myoung y colaboradores (2001) en su estudio sobre la recurrencia del queratoquiste, encontraron que la mayor tasa de reincidencia se daba en la quinta década de vida. ${ }^{14}$

Güler en el año 2012 realizó un estudio en el que se comparó la recurrencia de los OKC posterior a tres distintos tipos de tratamiento y no se encontró una diferencia estadísticamente significativa entre la enucleación simple, enucleación más irrigación con solución de Carnoy y la marsupialización de la lesión, ${ }^{13}$ concordando con la revisión realizada por Sharif y cols (2010) en la que se buscó comparar la efectividad de las diferentes intervenciones y acciones complementarias del tratamiento del queratoquiste, sin llegar a definir un tratamiento estadísticamente mejor que otro. ${ }^{9}$

El periodo de seguimiento aconsejado es una vez al año durante los primeros cinco años tras la eliminación de la lesión y posterior a este tiempo, un control cada dos años. ${ }^{16}$

Se recomienda el tratamiento temprano de estas lesiones explicando al paciente los pros y los contras de las distintas modalidades terapéuticas, así como también planificar y comprometer al paciente para mantener un control periódico, recalcando que las recidivas comúnmente pueden ocurrir a los cinco años después del tratamiento inicial, aunque se han reportado apariciones tardías hasta 40 años postratamiento.

CONFLICTO DE INTERESES: Los autores no manifiestan ningún conflicto de interés.

\section{Referencias Bibliográficas}

1 Wright JM, Vered M. Update from the 4th Edition of the World Health Organization Classification of Head and Neck Tumours: Odontogenic and Maxillofacial Bone Tumors. Head Neck Pathol. 2017;11(1):68-77.

2 Robles P, Roa I. Keratocystic odontogenic tumor: Clinicopathological aspects and treatment. J Oral Res. 2014;3(4):249-56.
3 Hyun HK, Hong SD, Kim JW. Recurrent keratocystic odontogenic tumor in the mandible: A case report and literature review. Oral Surgery, Oral Med Oral Pathol Oral Radiol Endodontology [Internet]. 2009;108(2):e7. Disponible en: http://dx.doi.org/10.1016/j.tripleo.2009.04.030

4 Rajendra Santosh AB. Odontogenic Cysts. Dent Clin North Am [Internet]. 2020;64(1):105-19. Disponible en: https://doi.org/10.1016/j.cden.2019.08.002

5 Kroll T, Streckbein P, Dreyer T, Bassaly B, Klußmann JP, Wittekindt C. İch kriege kaum noch Luft durch das linke Nasenloch". MMW - Fortschritte der Medizin. 2014;156(11):46-8.

6 Zhu F, Li X, Liu Z, He Y. An Odontogenic Keratocyst in the Temporal Region. J Craniofac Surg. 2019;30(8):2439-40.

7 Ruiz - García V. Tumor Odontogénico Queratoquístico Infectado: Características Imagenológicas Y Seguimiento: Reporte De Caso. Odontol Act Rev Científica. 2018;1(3):59-64.

8 Berge TI, Helland SB, Sælen A, Oren M, Johannessen AC, Skartveit L, et al. Pattern of recurrence of nonsyndromic keratocystic odontogenic tumors. Oral Surg Oral Med Oral Pathol Oral Radiol [Internet]. 2016;122(1):10-6. Available from: http://dx.doi.org/10.1016/j.oooo.2016.01.004

9 Sharif FNJ, Oliver R, Sweet C, Sharif MO. Interventions for the treatment of keratocystic odontogenic tumours. Cochrane Database Syst Rev. 2010;2010(9).

10 Cunha JF, Gomes CC, De Mesquita RA, Andrade Goulart EM, De Castro WH, Gomez RS. Clinicopathologic features associated with recurrence of the odontogenic keratocyst: A cohort retrospective analysis. Oral Surg Oral Med Oral Pathol Oral Radiol [Internet]. 2016;121(6):629-35. Dsiponible en: http://dx.doi.org/10.1016/j.oooo.2016.01.015

11 Zecha JAEM, Mendes RA, Lindeboom VB, Van Der Waal I. Recurrence rate of keratocystic odontogenic tumor after conservative surgical treatment without adjunctive therapies - A 35-year single institution experience. Oral Oncol [Internet]. 2010;46(10):740-2. Disponible en: http://dx.doi.org/10.1016/j.oraloncology.2010.07.004

2 Hashmi AA, Edhi MM, Faridi N, Hosein M, Khan M. Mutiple keratocystic odontogenic tumors (KCOT) in a patient with Gorlin syndrome: A case report with late presentation and absence of skin manifestations. BMC Res Notes. 2016;9(1):1-5.

13 Guler N, Şençift K, Demirkol Ö. Conservative management of keratocystic odontogenic tumors of jaws. Sci World J. 2012;2012.

14 Myoung $\mathrm{H}$, Hong SP, Hong SD, Lee J Il, Lim CY, Choung PH, et al. Odontogenic keratocyst: Review of 256 cases for recurrence and clinicopathologic parameters. Oral Surg Oral Med Oral Pathol Oral Radiol Endod. 2001;91(3):328-33.

15 Sembronio S, Albiero AM, Zerman N, Costa F, Politi M. Endoscopically assisted enucleation and curettage of large mandibular odontogenic keratocyst. Oral 
miento: Reporte De Caso. Odontol Act Rev Científica. 2018;1(3):59-64.

8 Berge TI, Helland SB, Sælen A, Oren M, Johannessen AC, Skartveit L, et al. Pattern of recurrence of nonsyndromic keratocystic odontogenic tumors. Oral Surg Oral Med Oral Pathol Oral Radiol [Internet]. 2016;122(1):10-6. Available from: http://dx.doi.org/10.1016/j.oooo.2016.01.004

9 Sharif FNJ, Oliver R, Sweet C, Sharif MO. Interventions for the treatment of keratocystic odontogenic tumours. Cochrane Database Syst Rev. 2010;2010(9).

10 Cunha JF, Gomes CC, De Mesquita RA, Andrade Goulart EM, De Castro WH, Gomez RS. Clinicopathologic features associated with recurrence of the odontogenic keratocyst: A cohort retrospective analysis. Oral Surg Oral Med Oral Pathol Oral Radiol [Internet]. 2016;121(6):629-35. Dsiponible en: http://dx.doi.org/10.1016/j.oooo.2016.01.015

11 Zecha JAEM, Mendes RA, Lindeboom VB, Van Der Waal I. Recurrence rate of keratocystic odontogenic tumor after conservative surgical treatment without adjunctive therapies - A 35-year single institution experience. Oral Oncol [Internet]. 2010;46(10):740-2. Disponible en: http://dx.doi.org/10.1016/j.oraloncology.2010.07.004

12 Hashmi AA, Edhi MM, Faridi N, Hosein M, Khan M. Mutiple keratocystic odontogenic tumors (KCOT) in a patient with Gorlin syndrome: A case report with late presentation and absence of skin manifestations. BMC Res Notes. 2016;9(1):1-5.

13 Guler N, Şençift K, Demirkol Ö. Conservative management of keratocystic odontogenic tumors of jaws. Sci World J. 2012;2012.

14 Myoung H, Hong SP, Hong SD, Lee J Il, Lim CY, Choung PH, et al. Odontogenic keratocyst: Review of 256 cases for recurrence and clinicopathologic parameters. Oral Surg Oral Med Oral Pathol Oral Radiol Endod. 2001;91(3):328-33.

15 Sembronio S, Albiero AM, Zerman N, Costa F, Politi M. Endoscopically assisted enucleation and curettage of large mandibular odontogenic keratocyst. Oral Surgery, Oral Med Oral Pathol Oral Radiol Endodontology [Internet]. 2009;107(2):193-6. Disponoble en: http://dx.doi.org/10.1016/j.tripleo.2008.07.020

16 Stoelinga PJW. The treatment of odontogenic keratocysts by excision of the overlying, attached mucosa, enucleation, and treatment of the bony defect with carnoy solution. J Oral Maxillofac Surg. 2005;63(11):1662-6.

17 Schmidt BL. The use of liquid nitrogen cryotherapy in the management of the odontogenic keratocyst. Oral Maxillofac Surg Clin North Am. 2003;15(3):393-405.

18 O'Neill R, Al-Hezaimi K. Identification of an odontogenic keratocyst and treatment with guided tissue regeneration: case report. J Can Dent Assoc [Internet]. 2011;77:b6. Disponible en: http://europepmc.org/abstract/MED/21385536text

19 Stoelinga PJW. Long-term follow-up on keratocysts treated according to a defined protocol. Int J Oral Maxillofac Surg. 2001;30(1):14-25.
Recibido: 03 de marzo de 2020

Aceptado: 21 de Abril de 2020 